\title{
A influência do Comportamento dos Usuários e das Operadoras de Telecomunicações em uma Rede Óptica Elástica
}

\author{
Vinicius Silva dos Anjos ${ }^{1}$, Rodrigo Campos Bortoletto ${ }^{1}$, Helio Waldman ${ }^{2}$ \\ ${ }^{1}$ Instituto Federal de Educação, Ciência e Tecnologia de São Paulo (IFSP) \\ 07115-000 - Guarulhos - SP - Brazil \\ ${ }^{2}$ Faculdade de Engenharia Elétrica e de Computação \\ Universidade Estadual de Campinas (Unicamp) - Campinas - SP - Brazil \\ anjos.viniciusealuno.ifsp.edu.br, bortolettodifsp.edu.br, \\ waldmand decom. fee. uni camp. br
}

\begin{abstract}
The increasing consumption of bandwidth for internet access and its communication services, which was exacerbated by the COVID-19 pandemic, requires studies on the influence of the collective behavior of users and operators on the dynamics of telecommunications networks. Key aspects such as: quality of service (QoS), connection cost, customer service experience, service rate, among others, are essential for operators to understand the needs of users, aiming to improve the relationship between operator and user. Thus, the objective of this work is to study the behavior of users and telecommunications operators, using the decision parameters to generate a simulation model that allows a realistic reproduction of the studied environment. Thus, the simulation model based on Multi-Agent Systems (MAS) was used together with a programming language for its modeling.
\end{abstract}

Resumo. O crescente aumento do consumo de banda para acesso à internet $e$ seus serviços para comunicação, que foi exacerbada pela pandemia de COVID19, demanda estudos sobre a influência do comportamento coletivo dos usuários e das operadoras na dinâmica das redes de telecomunicações. Aspectos chaves como: qualidade de serviço $(Q o S)$, custo da conexão, experiência de atendimento do consumidor, taxa de atendimento, entre outros, se mostram essenciais para que as operadoras compreendam as necessidades dos usuários, visando melhorar a relação entre operadora e usuário. Assim, o objetivo deste trabalho é estudar o comportamento dos usuários e das operadoras de telecomunicações, utilizando os parâmetros de decisão para gerar um modelo de simulação que permita reproduzir com realismo o ambiente estudado. Sendo assim, foi utilizado o modelo de simulação baseado em Sistemas Multiagentes (SMA) em conjunto com uma linguagem de programação para sua modelagem.

\section{Introdução}

As redes de telecomunicações constituem-se em um fator essencial na sociedade moderna. O crescimento do fluxo de dados e a mudança do comportamento dos usuários que passaram a consumir cada vez mais banda tem impacto direto nas operadoras de 
telecomunicações, as quais observam o crescimento deste tráfego e a necessidade de investimentos na expansão de suas redes. Portanto, o desenvolvimento de um adequado modelo de dimensionamento dessas redes torna-se importante à medida que influi diretamente em suas receitas [Rodriguez Castelan et al. 2020].

A abertura do mercado à concorrência e a consequente multiplicação das redes de telecomunicações, a probabilidade de bloqueio no atendimento de uma conexão deixa de ser um parâmetro adequado para contratação de serviços de infraestrutura, pois ela não é mais restrita ao monopólio de uma única empresa, passando a representar a possibilidade de bloqueio em uma ou mais operadora [Browning and Zupan 2020].

Em um ambiente competitivo com múltiplos prestadores de serviço (operadoras), a probabilidade de bloqueio do usuário não depende de uma única operadora: cada operadora sabe sua própria probabilidade de bloqueio, mas não tem ideia da probabilidade de bloqueio do usuário, isto é, a probabilidade do usuário ser bloqueado em todas as operadoras.

Somado a isso, os modelos atuais de dimensionamento de redes têm como objetivo a busca pela qualidade de serviço por meio da determinação de valores máximos de probabilidade de bloqueio. Por outro lado, este comportamento nem sempre vai ao encontro dos interesses dos usuários que buscam, na grande maioria das vezes, a minimização da probabilidade de bloqueio, e nem aos interesses das operadoras que buscam maximizar seus lucros [Scitovsky 2013].

Os Sistemas Multiagentes (SMA) são compostos por múltiplos elementos (agentes) computacionais interativos. Esses agentes possuem duas capacidades importantes: a de agir de forma autônoma, o que nesse cenário significa que o agente é capaz de decidir por si mesmo como agir para satisfazer seus objetivos, sem a intervenção humana ou de outros sistemas [Kaminski et al. 2016]; e a capacidade de interagir com outros agentes a partir de atividades sociais [Wooldridge 2009].

Em SMA, o comportamento inteligente depende das interações entre os agentes, o que significa que, uma vez que cada agente é autônomo, esse não pode forçar o outro a realizar ações ou alterar seu estado interno diretamente. Caso deseje que outro agente realize determinada tarefa, é necessário que haja comunicação entre eles. A capacidade de cooperação é chave fundamental para que a interação ocorra com êxito [Resch et al. 2017].

Este artigo tem por objetivo apresentar um estudo sobre a probabilidade de ocupação dos slots de espectro óptico em Redes Ópticas Elásticas em um cenário no qual duas operadoras de telecomunicações, ou seja, um duopólio, disputam requisições de usuários que buscam minimizar suas probabilidades de bloqueio.

A seção de materiais e métodos apresenta os cenários utilizados para o desenvolvimento do simulador, a seção de Resultados e Discussão trás os resultados obtidos do ambiente de simulação para o duopólio e no final são apresentadas as conclusões e trabalhos futuros.

\section{Redes Ópticas Elásticas}

As atuais redes de grade fixa são formadas por múltiplas portadoras ópticas espaçadas igualmente em frequência, formando uma grade padrão, cujo espaçamento corresponde à 
única granularidade disponível para acomodar as necessidades atuais de tráfego da rede [RECOMMENDATION 2012].

Uma consequência do uso da grade fixa em redes ópticas corresponde ao desperdício de banda devido a alocação de um comprimento de onda para conexões com baixa taxa de transmissão. A grade que acomodaria uma conexão com uma taxa de transmissão maior é desperdiçada devido à necessidade de acomodação desta requisição [Jinno et al. 2011, Christodoulopoulos et al. 2011].

As redes ópticas elásticas possuem uma grade de espectro flexível que concilia escalabilidade e flexibilidade na medida em que gera menor desperdício de banda para as demandas de baixa taxa de transmissão, também acomoda demandas por altas taxas de transmissão, e economiza a banda que seria desperdiçada caso essa mesma taxa de transmissão fosse dividida em uma rede óptica com grade fixa [Jinno et al. 2011, Christodoulopoulos et al. 2011].

O espectro das fibras ópticas é usado de forma flexível, assim pode ser obtido pelo uso de grades com menor espaçamento, em torno de 6,25 a $12,5 \mathrm{GHz}$, e pela possibilidade de união que permite o uso de porções contíguas do espectro.

\section{Materiais e Métodos}

A metodologia utilizada foi o desenvolvimento do ambiente de simulação e a coleta de dados com o intuito de realizar as simulações necessárias propostas no trabalho.

O ambiente foi modelado utilizando a linguagem Python e para o desenvolvimento do SMA foi escolhido o Python Agent DEvelopment framework (PADE) que é um framework para desenvolvimento, execução e gerenciamento de sistemas multiagentes em ambientes de computação distribuída [de Freitas et al. 2019].

Para o desenvolvimento da simulação foi criado um cenário de duopólio, onde existem duas empresas disputando os clientes em um mesmo ambiente, para tanto foram criados dois tipos de agentes: o agente empresa e o agente usuário. Abaixo será descrita a dinâmica de comportamento desses agentes.

Os agentes usuários são responsáveis por solicitarem requisições ao agente empresa sorteado. Já os agentes empresa são responsáveis por avaliar as requisições, podendo aceitar ou bloquear. A coleta de dados ocorre ao final das simulações, com as características principais atuantes na comunicação entre os agentes. Estas características estão expostas na Tabela 1.

Tabela 1. Características dos principais agentes atuantes na comunicação.

\begin{tabular}{l|l|l}
\hline Dados & Fonte & Objetivo \\
\hline Número do Agente & Agente Usuário & Identificar usuário da requisição \\
Tempo de vida & & Parâmetro analisado pelo agente empresa \\
Empresa & Agente Empresa & Empresa que aceitou a requisição \\
Estado & & $\begin{array}{l}\text { Estado da requisição (aceito ou bloqueado) } \\
\text { Entrada }\end{array}$ \\
\hline
\end{tabular}

O dado utilizado para a identificação da requisição é o Número do Agente e o critério de avaliação da requisição é o Tempo de Vida, que será inserido em um escalo- 
nador de eventos para posterior finalização da requisição, quando seu tempo de vida se esgotar. Os dados gerados após o processamento da requisição são Empresa, Estado e Entrada, com a finalidade de analisar a quantidade de requisições e a probabilidade de bloqueio.

Cada requisição poderá ser aceita por cada uma das operadoras presentes no duopólio, caso seja aceita pela operadora inicial, ou seja, a operadora sorteada para a requisição, será feita a contagem da requisição como aceita pela operadora primária, no caso a sorteada. Caso a requisição seja bloqueada pela operadora inicialmente sorteada, essa poderá ser aceita pela outra operadora, neste caso a requisição será considerada aceita pela operadora secundária.

A Figura 1 apresenta uma representação de um duopólio formado por duas empresas que competem pelos Agentes usuários, cada uma das redes de cada uma das operadoras é formada por um escalonador de eventos que armazena as requisições dos agentes usuários.

\section{Duopólio}

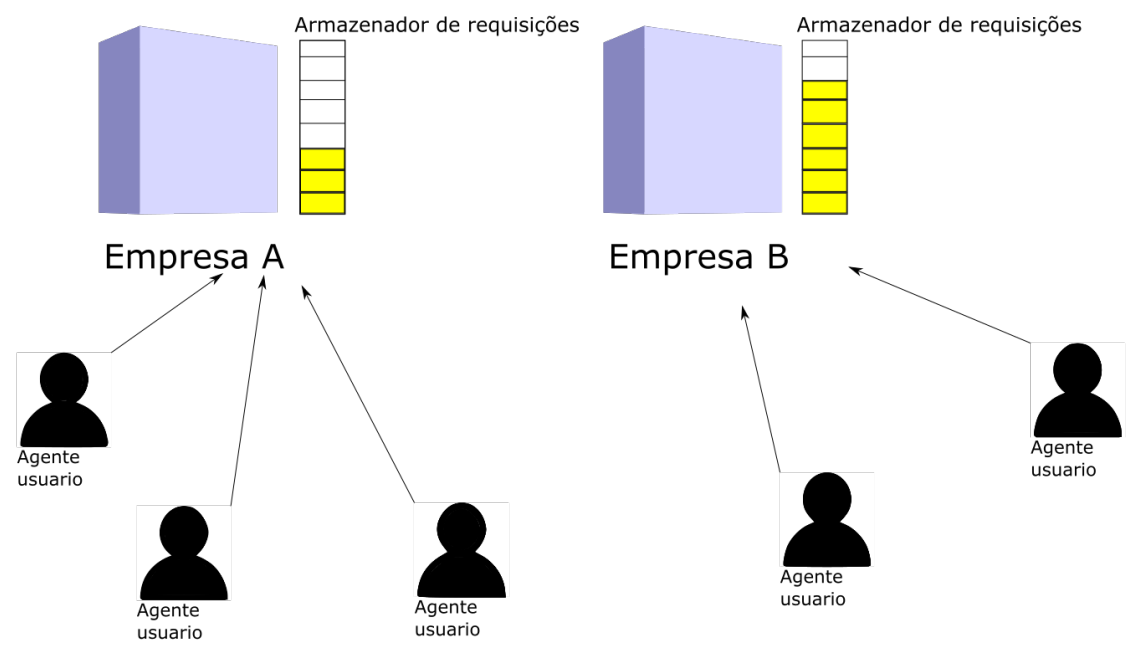

Figura 1. Representação visual do ambiente de simulação.

\section{Resultados e Discussão}

De acordo com as simulações, o fluxo de requisições de usuários é o principal fator resultante na saída das requisições aceitas ou bloqueadas.

Outro modo de afetar os resultados é aumentar o número de operadoras da simulação, com isso reduziria o número de bloqueios em comparação com a simulação de duopólio, neste caso originar-se-ia um oligopólio.

A Figura 2 apresenta as probabilidades de bloqueio obtidas na simulação para dois diferentes tempos entre requisições, observa-se que a partir de 400 requisições a probabilidade de bloqueio permanece constante, para todos os casos estudados. 


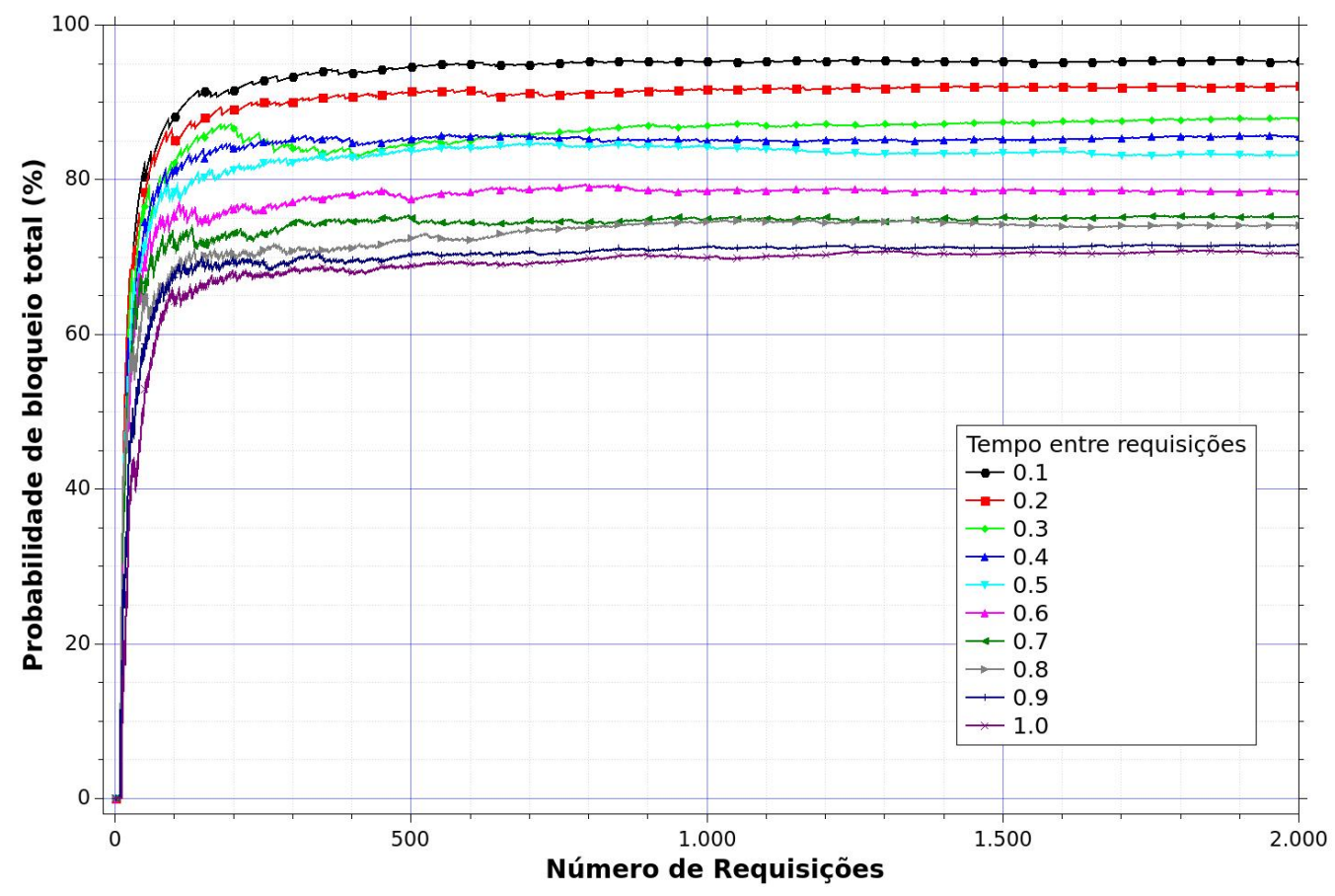

Figura 2. Gráfico com as probabilidade de bloqueio obtidas na simulação.

A Tabela 2 apresenta as probabilidades de bloqueio total para cada um dos tempos entre requisições. Observa-se que a medida que o tempo entre requisições aumenta a probabilidade de bloqueio vai reduzindo, demonstrando um comportamento coerente com o esperado para uma Rede óptica.

\section{Tabela 2. Probabilidade de bloqueio total para cada um dos tempos entre requisições}

\begin{tabular}{l|llllllllll}
\hline Tempo entre requisições & 0,1 & 0,2 & 0,3 & 0,4 & 0,5 & 0,6 & 0,7 & 0,8 & 0,9 & 1,0 \\
\hline Probabilidade de Bloqueio (\%) & 95 & 92 & 87 & 85 & 83 & 78 & 75 & 73 & 72 & 70 \\
\hline
\end{tabular}

\section{Conclusões}

A probabilidade total observada no gráfico não é constante, por se tratar de uma simulação, fazendo com que os valores apresentem variações. Para reduzir essa variação são necessárias múltiplas simulações com sementes diferentes para que seja possível obter a média da probabilidade de bloqueio para os dois casos.

Para os trabalhos futuros a função utilidade da simulação do SMA será alterada permitindo que fatores como: qualidade de serviço, custo da requisição, qualidade de atendimento da operadora, entre outros, possam ser levadas em consideração na escolha do agente usuário. Essas alterações permitirão análises mais apuradas de cada um dos fatores para o processo de escolha da operadora do usuário.

\section{Agradecimentos}

Os autores agradecem os suportes do projeto da Fundação de Amparo à Pesquisa do Estado de São Paulo (FAPESP) Proc. 2015/24341-7, bem como ao Programa Institucional 
de Bolsa de Iniciação Científica do IFSP (PIBIFSP) pela concessão de bolsa de estudo que possibilitou o desenvolvimento do projeto que originou o presente artigo.

\section{Referências}

Browning, E. K. and Zupan, M. A. (2020). Microeconomics: Theory and applications. John Wiley \& Sons.

Christodoulopoulos, K., Tomkos, I., and Varvarigos, E. A. (2011). Elastic bandwidth allocation in flexible ofdm-based optical networks. Journal of Lightwave Technology, 29(9):1354-1366.

de Freitas, B. K., Venturini, L. F., Domingues, M. A., da Rosa, M. A., and Issicaba, D. (2019). Exploiting pade to the simulation of multiagent restoration actions. In 2019 11th International Symposium on Advanced Topics in Electrical Engineering (ATEE), pages $1-5$. IEEE.

Jinno, M., Ohara, T., Sone, Y., Hirano, A., Ishida, O., and Tomizawa, M. (2011). Elastic and adaptive optical networks: Possible adoption s and future standardization aspects. IEEE Communications Magazine, 49(10).

Kaminski, N. J., Murphy, M., and Marchetti, N. (2016). Agent-based modeling of an iot network. In 2016 IEEE international symposium on systems engineering (ISSE), pages 1-7. IEEE.

RECOMMENDATION, G. (2012). Spectral grids for wdm applications: Dwdm frequency grid. International Telecommunications Union, .

Resch, M. M., Kaminski, A., and Gehring, P. (2017). The science and art of simulation I: Exploring-understanding-knowing. Springer.

Rodriguez Castelan, C., Malasquez, E. A., and Granguillhome, R. (2020). Welfare and competition.

Scitovsky, T. (2013). Welfare \& competition, volume 103. Routledge.

Wooldridge, M. (2009). An introduction to multiagent systems. John wiley \& sons. 